\title{
Efficiency of the balancing devices to power quality improve
}

\author{
Igor Naumov ${ }^{1,2, *}$, Sergey Podyachikh ${ }^{1}$, and Dmitriy Ivanov ${ }^{2}$ \\ ${ }^{1}$ Irkutsk State Agrarian University Named After A.A. Ezhevsky, Molodezhny Str., 1/1, Irkutsk \\ district, 664038 Molodezhny settlement, Russian Federation \\ ${ }^{2}$ Irkutsk National Research Technical University, Lermontov Str., 83, 664074 Irkutsk, Russian \\ Federation
}

\begin{abstract}
Violation of the symmetrical mode of operation of the electric network causes a deterioration in the quality of electric energy and significantly reduces the reliability of the functioning of electrical equipment and elements of the electric network. A mathematical model of an electrical network with a grounded neutral is proposed and a software package that allows us to evaluate the level of voltage asymmetry of a three-phase system depending on the changing unbalanced load was created. The simulation and calculation of the studied parameters for various combinations of loads and the technical means of balancing the operating mode of the electric network (the balancing device - BD) is carried out. Programs for the Matlab graphic editor are compiled and diagrams of changes in electricity quality indicators are constructed. A numerical analysis is carried out and recommendations are given to determine the most appropriate place for connecting the $\mathrm{BD}$ in the electrical network to improve the quality of electricity based on the calculation made.
\end{abstract}

\section{Introduction}

The growth of electricity consumption in rural areas has increased significantly due to the introduction of a large number of new electric receivers in residential areas, as well as new technologies in agricultural production. The operating modes of rural low-voltage distribution networks are objectively unbalanced. This is proven by numerous studies. The negative and zero voltage sequences have a very negative effect on electrical equipment. So, the negative sequence causes moments in engines with the opposite sign, which significantly affects the efficiency of the engine and its service life [1-2]. The zero sequence can lead to an abnormally large neutral bias voltage in normal modes and requires detailed consideration [3-10]. In addition, the zero sequence of currents creates additional losses of electricity, which not only increase its consumption, but also create conditions for the occurrence of fires [11].

In this regard, the creation of a mechanism for modeling asymmetric modes allows to analyze the use of symmetric devices to minimize the indicators of current and voltage

\footnotetext{
* Corresponding author: professornaumov@list.ru
} 
asymmetry. The purpose of the article is to study the technical possibilities of balancing the rural electric distribution networks operating modes on the $0,38 \mathrm{kV}$ electric network model.

\section{Materials and methods}

Two quality indicators characterize the asymmetry of the three-phase voltage system. These are the stress asymmetry coefficients for the negative $\left(\mathrm{K}_{2 \mathrm{U}}\right)$ and zero $\left(\mathrm{K}_{0 \mathrm{U}}\right)$ sequences. The different countries standards are set their own power quality values. For example, according to [12], the maximum permissible value of these coefficients should not exceed $4 \%$, and the normal value (for $95 \%$ of the measurement interval time) should not exceed $2 \%$. In addition, the network current asymmetry created by different types of unbalanced load leads to additional power losses characterized by a loss factor. This coefficient characterizes the excess of active power losses in the unsymmetric mode to the corresponding losses due to the flow of direct sequence currents $[1,11]$.

\section{Results}

The mathematical model of the $0.38 \mathrm{kV}$ electrical network to study asymmetric operating modes was used. This is shownin in the figure 1.

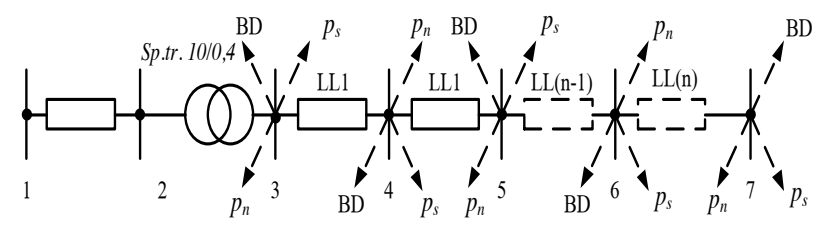

Fig. 1. Mathematical model of the system.

The system includes the following elements: 1-2 - medium voltage overhead power line. The length of the $10 \mathrm{kV}$ overhead line is adopted according to the standards of reliability of power supply and is equal to $16.7 \mathrm{~km}$ [1]. The complex resistance of the positive and negative sequences of this line, made by the AC 35 wire, is reduced to a voltage of $0.4 \mathrm{kV}$ and is equal to: $Z_{H V 1}=Z_{H V 2}=0,0243+j 0,01=0,0263 e^{j 22,37}$, Ohms; 2-3 - 10/0.4 kV power transformer with "Y/Y-n" winding connection scheme. The complex resistance of the positive, negative and zero sequences of the transformer depends on the rated power of the transformer. Consider the case when a transformer with a rated power of $S_{\text {nom. }}=100 \mathrm{\kappa VA}$ is used. For him, complex resistances positive and negative sequences are: $Z_{T 1}=Z_{T 2}=0,032+j 0,065=0,072 e^{j 64}$, Ohms, complex resistance of the zero sequences is $Z_{T 0}=0,254+j 0,582=0,635 e^{j 66,4}$ Ohms.; $3-0,4 \mathrm{kV}$ transformer busbars; $3-7-0.38 \mathrm{kV}$ line, $1 \mathrm{~km}$ long, made of self-supporting insulated wire SIP 1, with a cross section of $3 \times 50+1 \times 70 \mathrm{~mm}^{2}$. The line is divided into 4 equal sections of $0.25 \mathrm{~km}$ each. The resistances of each section of the positive (negative) sequence are equal: $Z_{I}=Z_{2}=0,641+j 0.0790=$ $0.6458 e^{j 7.0295}$, Ohms; complex resistance of the zero sequences $-Z_{0}=0,493+j 0.0685=$ $0.4977 e^{j 7.914}$ Ohms. In each of the nodes: 4, 5, 6 and 7, a three-phase unbalanced load: $p_{a}$, $p_{b}, p_{c}$ and a three-phase symmetric load $p_{s}$, are connected, whose value modules change in accordance with Table 1. 
Table 1. Changes the modules of three - phase unbalanced and symmetric loads.

\begin{tabular}{|c|c|c|c|c|}
\hline$p_{a}$ & $p_{b}$ & $p_{c}$ & $p_{s}$ & $p_{H}=p_{a}+p_{b}+p_{c}$ \\
\hline $\mathbf{0 , 0 1 7 7}$ & $\mathbf{0 , 0 0 4 2 5}$ & $\mathbf{0 , 0 0 3 0}$ & $\mathbf{0 , 2 2 5}$ & $\mathbf{0 , 0 2 5}$ \\
\hline $\mathbf{0 , 0 3 5 5}$ & $\mathbf{0 , 0 0 8 5 0}$ & $\mathbf{0 , 0 0 6 0}$ & $\mathbf{0 , 2 0 0}$ & $\mathbf{0 , 0 5 0}$ \\
\hline $\mathbf{0 , 0 5 3 2}$ & $\mathbf{0 , 0 0 8 5 0}$ & $\mathbf{0 , 0 0 9 1}$ & $\mathbf{0 , 1 7 5}$ & $\mathbf{0 , 0 7 5}$ \\
\hline $\mathbf{0 , 0 7 0 9}$ & $\mathbf{0 , 0 1 7 0 0}$ & $\mathbf{0 , 0 1 2 1}$ & $\mathbf{0 , 1 5 0}$ & $\mathbf{0 , 1 0 0}$ \\
\hline $\mathbf{0 , 0 8 8 7}$ & $\mathbf{0 , 0 2 1 2 5}$ & $\mathbf{0 , 0 1 5 1}$ & $\mathbf{0 , 1 2 5}$ & $\mathbf{0 , 1 2 5}$ \\
\hline $\mathbf{0 , 1 0 6 4}$ & $\mathbf{0 , 0 2 5 5 0}$ & $\mathbf{0 , 0 1 8 1}$ & $\mathbf{0 , 1 0 0}$ & $\mathbf{0 , 1 5 0}$ \\
\hline $\mathbf{0 , 1 2 4 1}$ & $\mathbf{0 , 0 2 9 8 0}$ & $\mathbf{0 , 0 2 1 1}$ & $\mathbf{0 , 0 7 5}$ & $\mathbf{0 , 1 7 5}$ \\
\hline $\mathbf{0 , 1 4 1 9}$ & $\mathbf{0 , 0 4 0 0 0}$ & $\mathbf{0 , 0 2 4 1}$ & $\mathbf{0 , 0 5 0}$ & $\mathbf{0 , 2 0 0}$ \\
\hline $\mathbf{0 , 1 5 9 6}$ & $\mathbf{0 , 0 3 8 3 0}$ & $\mathbf{0 , 0 2 7 2}$ & $\mathbf{0 , 0 2 5}$ & $\mathbf{0 , 2 2 5}$ \\
\hline $\mathbf{0 , 1 7 7 3}$ & $\mathbf{0 , 0 4 2 5 0}$ & $\mathbf{0 , 0 3 0 2}$ & $\mathbf{0 , 0 0 0}$ & $\mathbf{0 , 2 5 0}$ \\
\hline
\end{tabular}

It should be noted that the modules of $p_{a}, p_{b}, p_{c}$ are the ratio of capacity of single-phase loads in phases to the average full rated power three-phase unbalanced load and module $p_{s}$ - the ratio of active power symmetric load to its full capacity. The complexes of these values are given by using the arguments: $\varphi_{a}=\varphi_{b}=\varphi_{c}=25,24^{0} ; \varphi_{s}=36.87^{\circ} \cdot p_{a}+p_{b}+p_{c}=p_{n}$; $p_{n}+p_{s}=1$ - every time when the load are changes. Since the resistance of the negative sequence of a three-phase symmetric load (asynchronous motor) differs from the resistance of the positive sequence, a complex coefficient is used to determine it: $K_{2 s}=0,17+j 0,24=$ $0,0735 e^{j 54,689}[13]$. To each of the nodes $(4,5,6,7)$, and on the transformer busbars also (node 3), a BD can be connected. The BD parameters are defined in accordance with $[1,14]$ and equal: $Y_{B D 1}=Y_{B D 2}=0,0791 e^{j 90} ; Y_{B D O}=0,475 e^{j 0}$.

A method for determining the parameters of current and voltage asymmetry for the network model under study was developed for the network model under study. In detail, it was described in [1]. On the basis of this method, the software package "Asymmetry" was developed. The program is written in visual basic 6.0 using specialized libraries (MS Scripting Runtime, MS Excel 10.0 object library) and allows you to evaluate the asymmetry indicators at any point of the model under study with any changes in its parameters and any combination of symmetric, asymmetric loads and BD. Based on the calculation of the current and voltage asymmetry indicators according to the "Asymmetry" program, the values of the changing coefficients of the reverse and zero voltage sequences in each of the load nodes are obtained. At the same time, the possibility of including the BD in each of the nodes, and in its absence, was considered. According to the obtained calculations, diagrams of changes in these coefficients depending on the changing three-phase unbalanced load are constructed.

Let us consider how the electric power quality indicators that characterize the asymmetry of a three-phase voltage system (the coefficients of asymmetry in the negative and zero sequence) change in the load nodes and on the buses of a transformer substation (Figures 2-7). 


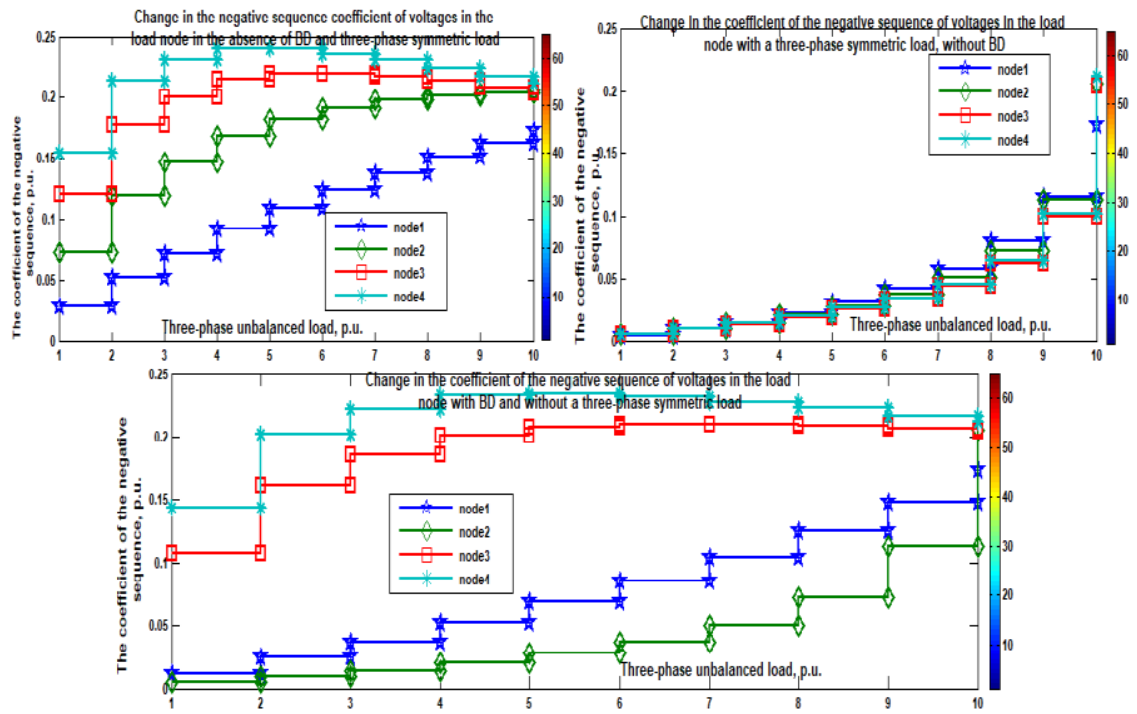

Fig. 2. Diagram of the change in the negative sequence coefficient of the voltage in the load nodes with different combinations of three-phase unbalanced load and BD.

The values of the indicators were studied in each load node $\left(\mathrm{K}_{2 \mathrm{U}}\right.$ and $\left.\mathrm{K}_{0 \mathrm{U}}\right)$, as well as on the power transformer buses (TB) with the following combinations of three-phase symmetric $\left(p_{s}\right)$ load and balancing device $(\mathrm{BD}): 1-\mathrm{BD}$ and $p_{s}$ are missing in all nodes and on the transformer busbars (TB); $2-p_{s}$ is enabled in all four nodes, $\mathrm{BD}$ is absent; $3-\mathrm{BD}$ is enabled on the transformer buses, $p_{s}$ is absent in all nodes; 4- BD is enabled on transformer buses, $p_{s}$ is enabled in each of the 4 nodes; $5-p_{s}$ is enabled in all four load nodes, BD is enabled in 1 node; $6-p_{s}$ is enabled in all four load nodes, BD is enabled in 2 node; $7-p_{s}$ is enabled in all four load nodes, BD is enabled in 3 node; $8-p_{s}$ is enabled in all four load nodes, BD is enabled in 4 node. The results of changes in the studied coefficients are as follows.

With 1 combination of $\mathrm{BD}$ and $p_{s}$, the average value of the coefficients of the negative and zero sequence in the first load node $\left(\mathrm{K}_{2 \mathrm{U}}, \mathrm{K}_{0 \mathrm{U}}\right)$ is 0.0812 and 0.1010 , respectively; on $\mathrm{TB}$, these coefficients are 0.0063 and 0.0564 . This difference in values is explained by the fact that in an electric network with a predominant utility load (single-phase electric receivers), the negative sequence of currents and voltages is minimal. For the second node $\mathrm{K}_{2 \mathrm{U}}, \mathrm{K}_{0 \mathrm{U}}$ are: 0.1530 and 0.1266 ; for the third node -0.2074 and 0.1675 ; for the fourth node - $0.2168 ; 0.1923$. As can see, there is a gradual increase of the indicators values in each of the load nodes, compared with the value of these indicators on the TB, namely: in the first load node, the value of $\mathrm{K}_{2 \mathrm{U}}$ is 12.9 times, $\mathrm{K}_{0 \mathrm{U}}$ is 1.79 times; in the second node, these indicators increase in 24.3 and 2.3 times; in the third - 32.9 and 2.97 times, and in the fourth -34.4 and 3.4 times. In combination 2, the inclusion of a three-phase symmetric load should have a certain effect. Let's analyze how this manifests itself in the results of calculations. On the TB, the coefficients $\mathrm{K}_{2 \mathrm{U}}$ and $\mathrm{K}_{0 \mathrm{U}}$ are: 0.012 and 0.0504 ; in the first node -0.0175 and 0.1106 ; in the second -0.0175 and 0.1144 ; in the third -0.0164 and 0.1299 and in the fourth- 0.0167 and 0.1159 , respectively. Thus, compared to the 1 combination, the $\mathrm{K}_{2 \mathrm{U}}$ coefficient on the tires increased by 1.9 times. This is due to the fact that the motor load (and this is $p_{s}$ ) increases the negative sequence of stresses, but its symmetrical effect is obvious since $\mathrm{K}_{0 \mathrm{U}}$ has decreased on $11 \%$. In the 1 node of the load, $\mathrm{K}_{2 \mathrm{U}}$ is reduced in 4.64 times, in the second - also, in the third - in 4.95 times and in the 
fourth-in 4.86 times, compared to the value of this indicator in the corresponding nodes for 1 combination. The $\mathrm{K}_{0 \mathrm{U}}$ coefficient in the first node increased on $9 \%$, in the second decreased on $11 \%$, in the third - on $29 \%$, in the fourth - on $66 \%$. For the remaining six combinations of $\mathrm{BD}$ and $p_{s}$, we will compare the average value of the studied indicators for 4 load nodes at once.
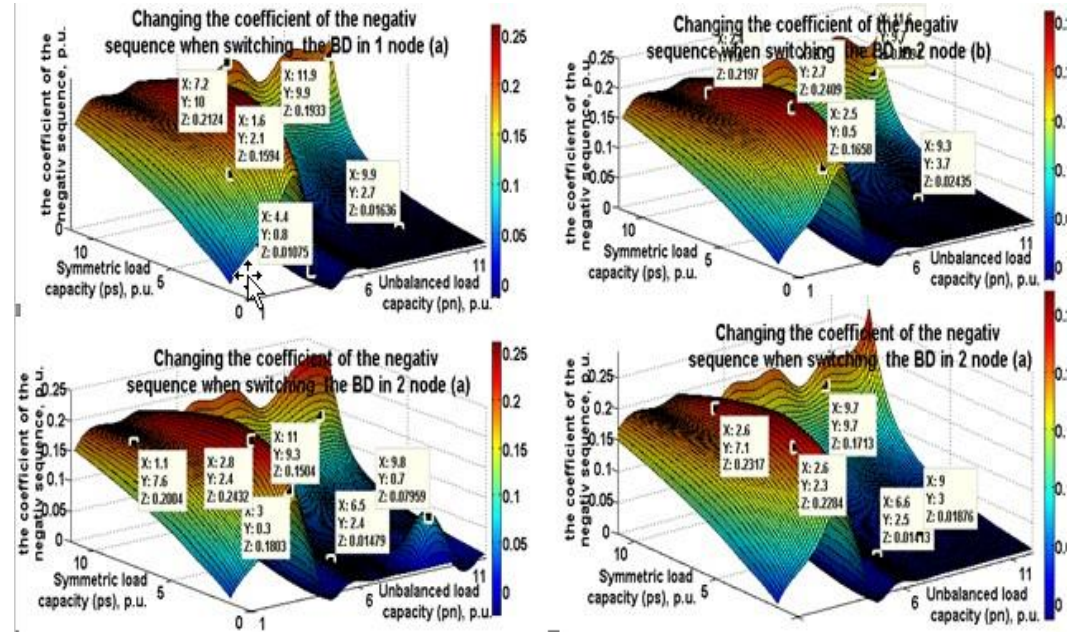

Fig. 3. Diagrams of the change in the coefficient of the negative sequence of stresses in the load nodes with different combinations of $p s$ and $\mathrm{BD}$.

When switching off the three-phase symmetric load from all 4 nodes and switching the $\mathrm{BD}$ on the TB (3 combination), the average value of $\mathrm{K}_{2 \mathrm{U}}$ and $\mathrm{K}_{0 \mathrm{U}}$ on the TB was 0.0033 and 0.0537 , respectively. At the same time, the average values of these coefficients for all four nodes were 0.1008 and 0.1625 , that is, the indicators increased in the nodes in average in 30.5 and 3 times.

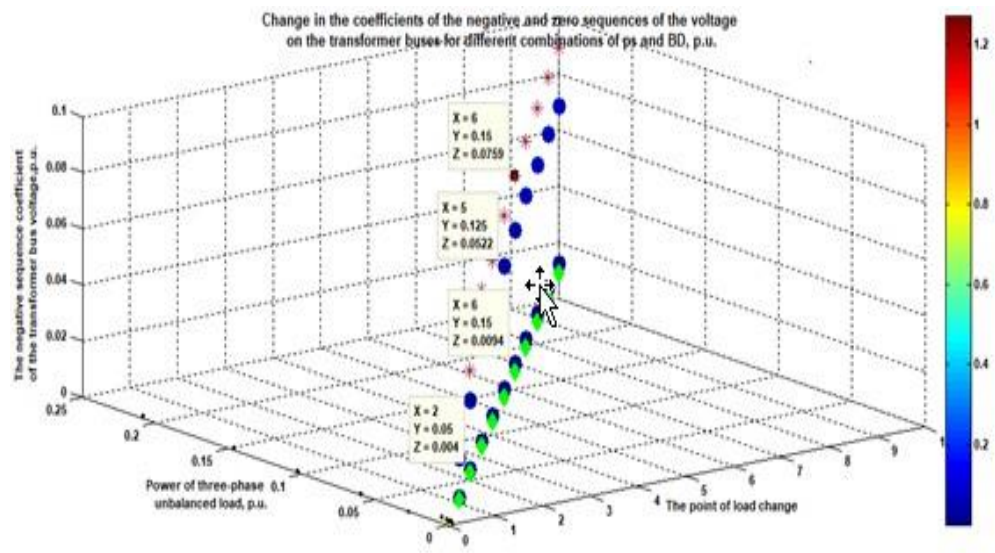

Fig. 4. Change in the coefficients of the negative and zero voltage sequences on the transformer buses in the absence of a three-phase symmetric load in the nodes and switching on (off) BD on the buses.

When switching off the three-phase symmetric load from all 4 nodes and switching the $\mathrm{BD}$ on the TB (3 combination), the average value of $\mathrm{K}_{2 \mathrm{U}}$ and $\mathrm{K}_{0 \mathrm{U}}$ on the TB was 0.0033 and 0.0537 , respectively. At the same time, the average values of these coefficients for all four nodes were 0.1008 and 0.1625 , that is, the indicators increased in the nodes in average in 30.5 and 3 times. With 4 combinations, the $\mathrm{BD}$ is connected on the $\mathrm{TB}$, and the $p_{s}$ is 
connected to each of the load nodes. In this case, the values of $\mathrm{K}_{2 \mathrm{U}}$ and $\mathrm{K}_{0 \mathrm{U}}$ on the TB are 0.0023 and 0.0388 . As can be seen, the BD allowed to reduce the value of these indicators (compared to the 3 combination) on 44 and $38.4 \%$, respectively. In the load nodes, the $\mathrm{K}_{2 \mathrm{U}}$ coefficient remained virtually unchanged, while $\mathrm{K}_{0 \mathrm{U}}$ decreased on $13.6 \%$. As shown above 5, 6, 7 and 8 combinations are characterized by connection the $p_{s}$ in each of the 4 nodes and alternate connection the $\mathrm{BD}$ in each node. In the 5 combination of $\mathrm{K}_{2 \mathrm{U}}$ and $\mathrm{K}_{0 \mathrm{U}}$ on the $\mathrm{TB}$ are equal to 0.0009 and 0.0012 , and in the nodes on average 0.0178 and 0.1079 , respectively. In this case, the indicators are reduced in 2.56 and 32.3 times on the TB and in 5.7 and 1.33 times in the load nodes. 6 the combination is characterized by the value of the indicators $\mathrm{K}_{2 \mathrm{U}}$ and $\mathrm{K}_{0 \mathrm{U}}$ equal, respectively: on the TB - 0.0012 and 0.0509 and in the nodes -0.0180 and 0.1083 . In this case, the indicators on the TB increased: $\mathrm{K}_{2 \mathrm{U}}$ on $33 \%, \mathrm{~K}_{0 \mathrm{U}}-$ in 42.4 times, and in the load nodes, the indicators practically did not change. The inclusion of BD 3 and 4 nodes (the combination of 7 and 8 , respectively) practically does not influence the indicators on the TB and change $\mathrm{K}_{2 \mathrm{U}}$ in the load nodes. Only the $\mathrm{K}_{0 \mathrm{U}}$ index decreases slightly - in the 7th combination it decreases in 1.14 times, in the 8 -th - in 1.36 times, compared to the 6-th combination.

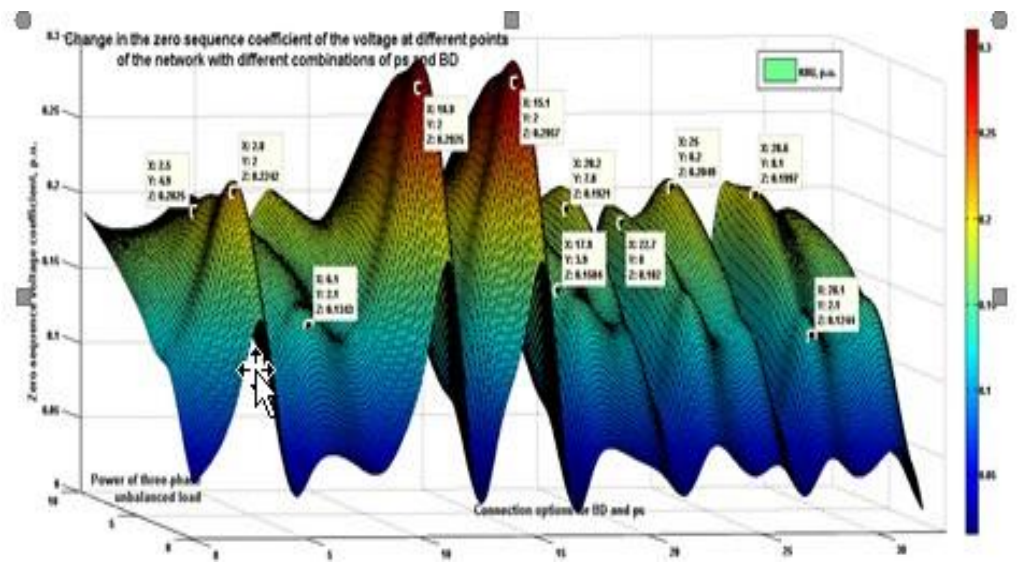

Fig. 5. Diagram of the change in the zero sequence coefficient of the voltage at different points of the network with different combinations of ps and BD.

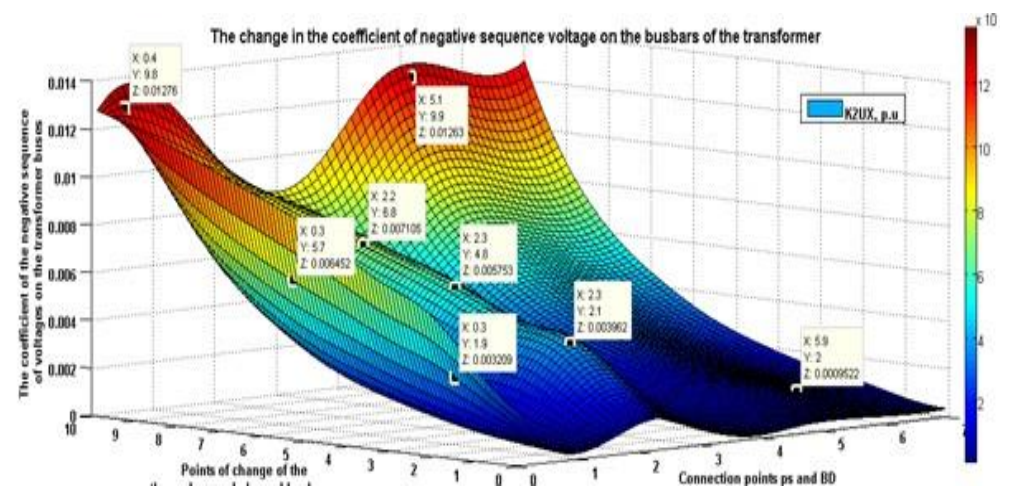

Fig. 6. Change the coefficient negative sequence of voltage on the transformer buses, when switching on the ps and BD in different load nodes. 


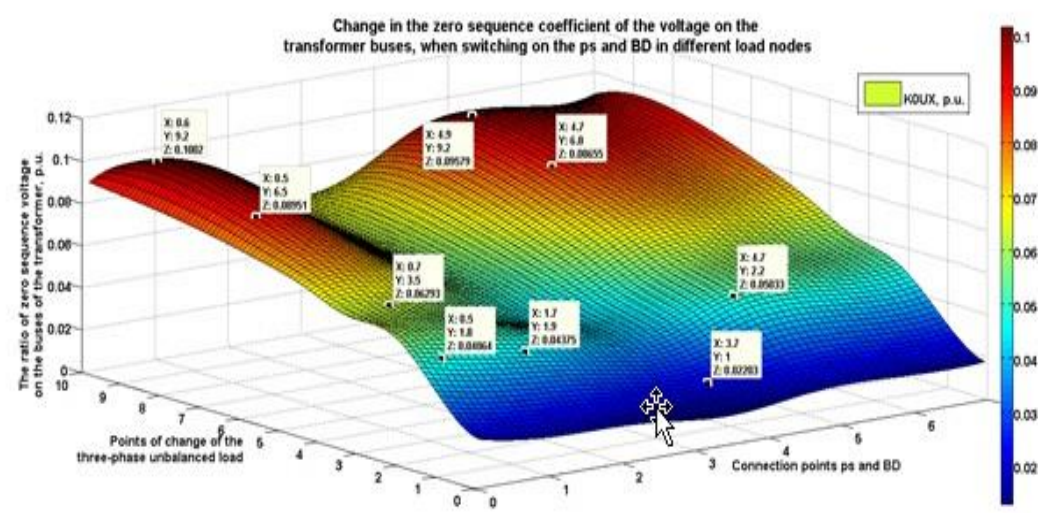

Fig. 7. Change the coefficient zero sequence of voltage on the transformer buses, when switching on the ps and BD in different load nodes.

\section{Conclusions}

- The balancing device, has a significant effect on reducing the indicators that characterize the asymmetry of stresses.

- The greatest decrease in the coefficients of the reverse and zero sequence of voltage in any load node or on the transformer buses (on average, more than 5 times) is observed in the case when the BD is switched on in this load node.

\section{Reference}

1. I. V. Naumov, Reducing losses and improving the quality of electric energy in rural distribution networks of $0.38 \mathrm{kV}$ with the help of symmetric devices, 387 (2002)

2. I. V. Naumov, N. V. Savina, M. V. Shevchenko, E3S Web of Conferences, 58, 1 (2018)

3. L. V. Fetisov, N. V. Rozhentsova, O. A. Bulatov, News of Higher Educational Instituti ons, 11(12), 99 (2018)

4. A. Girshin, V. Goryunov, E. Kuznetsov, D. Safonov, MATEC Web of Conferences, 70, 1 (2016)

5. D. E. Dulepov, T. E. Kondranenkova, the VIII International Scientific and Technical Conference "Electric power engineering through the eyes of youth" 328 (Samara: Samara State Technical University, 2017)

6. M. Zadeh, M. Sanaye-Pasand, A. Kadivar, IEEE Trans. Power Del., 23, 2472 (2008)

7. M. I. Zhilin, O. V. Vorkunov, Improving the quality of electric energy, The priority directions of development of science, 83 (2017)

8. L. Zjavka, V. Snášel, Elect. Power Syst. Res., 137, 113 (2016)

9. I. Kholiddinov, Electric Power Quality Analysis 6-10/0.4 kV, Distribution Networks Energy and Power Engineering, 8, 263 (2016)

10. I. V. Naumov, International Science and Technology Conference "EastConf" INSPEC 1 (2019)

11. Electrical energy, Compatibility of technical means is electromagnetic, Standards for the quality of electrical energy in general - purpose power supply systems, https://ww 
w.elec.ru/

12. S. A. Kulagin, Resistance of the reverse sequence of the agricultural load node, 18 (1980)

13. I. V. Naumov, E. A. Belousova, Vestnik Krasgau, 1, 99 (2017) 